\title{
POSTERS
}

\section{Turbulent Outflows from [WC] Nuclei}

\author{
Y. Grosdidier ${ }^{1,2,3}$, A. Acker ${ }^{2}$, A.F.J. Moffat ${ }^{1,3}$, O. Chesneau ${ }^{2}$ and T. Dimeo ${ }^{2}$ \\ ${ }^{1}$ Université de Montréal; ${ }^{2}$ Observatoire de Strasbourg; ${ }^{3}$ Observatoire du mont Mégantic
}

In the framework of the extensive study of [WC]-type nuclei of planetary nebulae undertaken since 1993 by Acker and collaborators, we report wind fluctuations for the two brightest late-type [WC] stars in the sky, observed at Observatoire de Haute-Provence (OHP, France) and Observatoire du mont Mégantic (OMM, Canada). In particular we confirm Balick et al.'s (1996, AJ, 111, 834) CIII $\lambda 5696$ line profile variations for the [WC8] nucleus of NGC 40. Moving features seen on the top of this line are interpreted as outflowing "blobs" which are accelerated in the WR wind. Kinematic parameters have been derived and compared to those observed for massive WR stars. This will be discussed in a forthcoming paper (Grosdidier et al., 1996, in preparation). Assuming the average motion of the blobs reflects the global wind expansion and adopting the ad hoc $\beta$ velocity law, we find that the exponent $\beta$ must be larger than 3 in order to fit the measured radial accelerations (up to $\sim 0.08 \mathrm{~km} \mathrm{~s}^{-2}$ ). Evidence for clumping in the wind of the [WC9] nucleus $\mathrm{BD}+30^{\circ} 3639$ is shown for the first time and presented in Acker et al. (1996, A\&ALetters, in press). In contrast to NGC 40, the clumps in this star show very low acceleration.

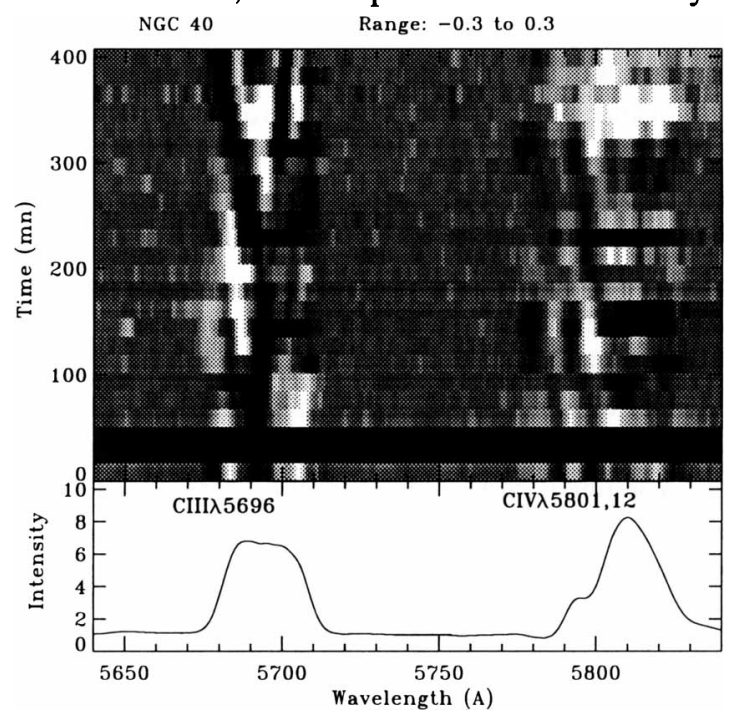

NGC 40: Grayscale plot of differences from the mean profile for the CIII $\lambda 5696$ and CIV $\lambda \lambda 5801,12$ lines as a function of time for one night's observing (OMM, 1996 January 15; time elapses from UT=23:53). 